\title{
Adaptive Bayesian inference in the Gaussian sequence model using exponential-variance priors
}

\author{
Debdeep Pati \\ Department of Statistics, Florida State University, Tallahassee, FL, \\ email:debdeep@stat.fsu.edu \\ Anirban Bhattacharya \\ Department of Statistics, Texas A $8 M$ University, College Station, TX, \\ email:anirbanb@stat.tamu.edu
}

\begin{abstract}
We revisit the problem of estimating the mean of an infinite dimensional normal distribution in a Bayesian paradigm. Of particular interest is obtaining adaptive estimation procedures so that the posterior distribution attains optimal rate of convergence without the knowledge of the true smoothness of the underlying parameter of interest. Belitser \& Ghosal [1] studied a class of power-variance priors and obtained adaptive posterior convergence rates assuming that the underlying smoothness lies inside a countable set on which the prior is specified. In this article, we propose a different class of exponential-variance priors, which leads to optimal rate of posterior convergence (up to a logarithmic factor) adaptively over all the smoothness levels in the positive real line. Our proposal draws a close parallel with signal estimation in a white noise model using rescaled Gaussian process prior with squared exponential covariance kernel.

Keywords: adaptive; Bayesian; Gaussian process; posterior contraction; sequence model.
\end{abstract}

\section{Introduction}

We consider the infinite Gaussian sequence model

$$
X_{i}=\theta_{i}+\epsilon_{i}, \quad \epsilon_{i} \sim \mathrm{N}\left(0, \sigma^{2}\right), \quad i=1,2, \ldots,
$$

Preprint submitted to Journal of ${ }^{A} T_{E} X$ Templates

March 31, 2015

(C) 2015. This manuscript version is made available under the Elsevier user license http://www.elsevier.com/open-access/userlicense/1.0/ 
where the parameter of interest is the infinite-vector $\boldsymbol{\theta}=\left(\theta_{1}, \theta_{2}, \ldots\right) \in \ell_{2}$, i.e., $\sum_{i=1}^{\infty} \theta_{i}^{2}<\infty$. Model (1) has received widespread attention since it encapsulates many intrinsic conceptual issues associated with non-parametric estimation. In particular, model (1) is equivalent to the canonical signal-in-white-noise model

$$
X(t)=\int_{0}^{t} f(s) d s+\sigma W(t), \quad t \in[0,1]
$$

where $f \in L_{2}[0,1]$ is the unknown function to be estimated based on noisy measurements $X(t), t \in[0,1]$, and $W(\cdot)$ is a standard Wiener process. The equivalence between (2) and (1) can be established by considering an orthonormal basis $\left\{\psi_{i}\right\}$ of $L_{2}[0,1]$ (for example, the Fourier basis) with the standard inner product $\langle g, h\rangle=\int_{t=0}^{1} g(t) h(t) d t$, and setting $X_{i}=\left\langle X, \psi_{i}\right\rangle, \theta_{i}=\left\langle f, \psi_{i}\right\rangle$ and $\epsilon_{i}=\left\langle W, \psi_{i}\right\rangle$; see, for example, Chapter 1 of Tsybakov [2]. With the calibration $\sigma=1 / \sqrt{n}$ in (1), Pinsker [3] established the minimax quadratic risk of estimating $\boldsymbol{\theta}$ over Sobolev ellipsoids of the form $\Theta_{\beta}(B)=\left\{\boldsymbol{\theta}: \sum_{i=1}^{\infty} i^{2 \beta} \theta_{i}^{2} \leq B\right\}$ as

$$
\inf _{\hat{\boldsymbol{\theta}}} \sup _{\boldsymbol{\theta} \in \Theta_{\beta}(M)} \mathbb{E}_{\boldsymbol{\theta}}\|\hat{\boldsymbol{\theta}}-\boldsymbol{\theta}\|_{2}^{2} \asymp n^{-2 \beta /(2 \beta+1)},
$$

where $\mathbb{E}_{\boldsymbol{\theta}}$ denotes an expectation with respect to the distribution of $\mathbf{X}=$ $\left(X_{1}, X_{2}, \ldots\right)$ given $\boldsymbol{\theta}$. Note that the minimax risk is the same as in the case of estimating a $\beta$-smooth function [4]. Adaptive estimation strategies without 5 the knowledge of the "smoothness"-parameter $\beta$ were first developed by [5]. We refer the reader to the monograph by Johnstone [6] which provides an excellent background \& introduction to the infinite sequence model and reviews minimax and adaptive estimation in this context.

In a Bayesian framework, Zhao 7] considered independent Gaussian priors on the entries of $\boldsymbol{\theta}$ of the form $\theta_{i} \sim \mathrm{N}\left(0, \tau_{i}^{2}\right)$ with $\tau_{i}^{2}=\tau_{i}^{2}(\beta)=i^{-(2 \beta+1)}$. The resulting posterior mean was shown to attain the minimax rate (3) when the true $\boldsymbol{\theta}_{0} \in \Theta_{\beta}$. For the same prior, Belitser \& Ghosal [1] established that the posterior contraction rate $[8$ coincides with the minimax rate, i.e., the posterior probability assigned to an $\ell_{2}$ neighborhood of the true parameter having radius 15 a constant multiple of the minimax rate converges to one almost surely. 
An unappealing aspect of the the above prior is that it requires the knowledge of the true smoothness parameter $\beta$. The main contribution of Belitser \& Ghosal [1] was to develop an adaptive Bayesian procedure which attains the minimax rate without knowledge of the smoothness parameter. Bayesian procedures offer a natural prescription for adaptation by introducing one or more additional level of hierarchy in defining the prior. Rather than choosing a fixed level of the smoothness $\beta$, Belitser \& Ghosal [1] considered a discrete prior on $\beta$ and showed that the resulting hierarchical procedure adapts to any smoothness level in the prior support. Choosing the discrete set to be a dense subset of the continuum (e.g., the set of rationals), it was additionally shown that one could adapt to any $\beta>0$, though the posterior contraction rate overshot the minimax rate by an arbitrarily small positive power of $n$ in this case.

In this paper, we propose a class of exponential-variance priors on $\boldsymbol{\theta}$ which is fundamentally different from the power-variance priors studied in the aforementioned literature [7, 1]. The proposed class of priors is indexed by a positive parameter $a$, which plays a similar role as an inverse-bandwidth parameter in nonparametric kernel estimation [2]. We first consider a non-adaptive scenario where an optimal choice of the parameter $a$ given the knowledge of $\beta$ is discussed. We next show that for a large class of prior distributions on $a$, the posterior 35 achieves the minimax rate (up to a logarithmic term) for $\boldsymbol{\theta}_{0} \in \Theta_{\beta}$ for any $\beta>0$. Since the prior distribution does not require knowledge of $\beta$, the procedure is fully adaptive. Finally, we provide a heuristic argument to relate our prior with the rescaled Gaussian process priors developed by van der Vaart \& van Zanten 9, 10 for nonparametric function estimation. This connection may be helpful 40 in extending the results proven in this paper for the infinite sequence model to Gaussian process regression and related settings.

\section{Preliminaries}

Let $\ell_{2}=\left\{\boldsymbol{\theta}=\left(\theta_{1}, \theta_{2}, \ldots\right): \sum_{i=1}^{\infty} \theta_{i}^{2}<\infty\right\}$ denote the space of squaresummable sequences. We shall write $\|\cdot\|$ for the $\ell_{2}$ norm throughout the paper, 
45 so that for any $\boldsymbol{\theta} \in \ell_{2},\|\boldsymbol{\theta}\|^{2}=\sum_{i=1}^{\infty} \theta_{i}^{2}$. Let $\Theta_{\beta}=\left\{\boldsymbol{\theta} \in \ell_{2}: \sum_{i=1} i^{2 \beta} \theta_{i}^{2}<\infty\right\}$ denote the Sobolev space of infinite dimensional vectors with "smoothness" $\beta>0$, and define the Sobolev norm $\|\theta\|_{\beta}=\left(\sum_{i=1} i^{2 \beta} \theta_{i}^{2}\right)^{1 / 2}$. Finally, let $\Theta_{\beta}(B)$ denotes a Sobolev-ball of radius $\sqrt{B}$ defined as $\left\{\boldsymbol{\theta} \in \Theta_{\beta}:\|\theta\|_{\beta}^{2}<B\right\}$.

Let $\mathbf{X}$ denote the infinite-dimensional random vector $\mathbf{X}=\left(X_{1}, X_{2}, \ldots\right)$ distributed as (1); we shall use $P_{\boldsymbol{\theta}}$ to denote the distribution of the $\mathbf{X}$. The notations $\mathbb{E}_{\theta} g(X) / \mathbb{V}_{\theta} g(X)$ are used to denote the expectation/ variance of $g(X)$ with respect to the distribution $P_{\theta}$ of $\mathbf{X}$. Define for $\epsilon>0$ and $\boldsymbol{\theta}_{0} \in \ell_{2}$, the Kullback-Leibler (KL) neighborhood of $P_{\boldsymbol{\theta}_{0}}$ as

$$
\mathcal{K}\left(P_{\boldsymbol{\theta}_{0}} ; \epsilon\right)=\left\{\boldsymbol{\theta}: \int P_{\boldsymbol{\theta}_{0}} \log \left(P_{\boldsymbol{\theta}_{0}} / P_{\boldsymbol{\theta}}\right)<\epsilon^{2}, \int P_{\boldsymbol{\theta}_{0}}\left[\log \left(P_{\boldsymbol{\theta}_{0}} / P_{\boldsymbol{\theta}}\right)\right]^{2}<\epsilon^{2}\right\} .
$$

Let $L_{2}[0,1]$ denote the space of square integrable functions on $[0,1]$. To distinguish from the $\ell_{2}$ norm, let $\|\cdot\|_{2}$ denote the $L_{2}$ norm on $[0,1]$ with respect to the Lebesgue measure. Throughout $C, C^{\prime}, C_{1}, C_{2}, \ldots$ are generically used to denote positive constants whose values might change from one line to another, but are independent from everything else. $\lesssim / \gtrsim$ denote inequalities upto a constant multiple. $a \asymp b$ when we have both $a \lesssim b$ and $a \gtrsim b$.

${ }_{55}$ Let $\phi(t)=(2 \pi)^{-1 / 2} \exp \left(-t^{2} / 2\right)$ denote the standard normal density and let $\phi_{\sigma}(t)=(1 / \sigma) \phi(t / \sigma)$.

\section{Prior specification and main results}

In this Section, we propose a class of exponential-variance priors on $\ell_{2}$ and state our results on posterior concentration using such priors. Proofs of all results are deferred to a supplemental document.

Consider the infinite sequence model (1) with $\sigma=1 / \sqrt{n}$. We define a class of exponential-variance priors on $\boldsymbol{\theta}$ as follows:

$$
\theta_{i} \mid a \sim \mathrm{N}\left(0, \tau_{i}^{2}(a)\right), \quad \tau_{i}^{2}(a)=\frac{1}{a} e^{-i / a}, \quad i=1,2, \ldots
$$

We shall use $\Pi_{a}$ to denote the prior (4) on $\boldsymbol{\theta}$ and denote the corresponding posterior by $\Pi_{a}(\cdot \mid \mathbf{X})$. For the class of power-variance priors with prior variance 
$\mathbb{V}\left(\theta_{i}\right)=i^{-(2 \beta+1)}$, the priors draws almost surely belong to $\Theta_{\alpha}$ for any $\alpha<\beta$. However, it is easy to see that for any fixed value of $a$, prior draws from the



Figure 1: Plot of $\tau_{i}^{2}(a)$ in 4 against $i$ for 3 different values of $a$. With increasing $a$, the rate of decay is slowed down.

proposed exponential-variance prior lie in any $\Theta_{\alpha}$ with probability one. This might seemingly suggest that the proposed prior is not useful in settings where the true $\boldsymbol{\theta}_{0} \in \Theta_{\beta}$ for some finite $\beta$. However, we show in Theorem 3.1 below that the deterministic choice $a=a_{n}=n^{1 /(2 \beta+1)}$ leads to the posterior contracting at the minimax rate (up to a logarithmic term) over $\Theta_{\beta}(B)$. Intuitively, larger values of $a$ produce rougher draws as can be seen from the fact that $\mathbb{E}\|\boldsymbol{\theta}\|_{\beta}^{2}$ is an increasing function of $a$. This is further illustrated in Figure 1 where a plot of $\tau_{i}^{2}(a)$ against the index $i$ is provided for three different values of $a$. It is evident that although the decay of $\tau_{i}^{2}(a)$ is exponential in $i$ for any fixed $a$, the effective rate of decay slows down for larger values of $a$, thereby making the resulting prior more appropriate for less smooth truths. The choice $a_{n}=n^{1 /(2 \beta+1)}$ achieves the correct bias-variance tradeoff. Note that the typical bandwidth choice for a $\beta$-smooth function in kernel estimation is $n^{-1 /(2 \beta+1)}$; see for example, Chapter 1 of [2]. Therefore, the parameter $a$ in the exponential-variance prior plays the role of an inverse-bandwidth parameter rather than directly indexing the so smoothness as in case of the power-variance priors. The proof of Theorem 3.1 
can be found in the supplemental document.

Theorem 3.1. Consider model (1) with $\sigma=1 / \sqrt{n}$ and assume the true datagenerating parameter $\boldsymbol{\theta}_{0} \in \Theta_{\beta}$. Let the prior on $\boldsymbol{\theta}$ be given by $\Pi_{a}$ as in (4), with $a \equiv a_{n}=n^{1 /(2 \beta+1)}$. Then, for any sequence $M_{n} \rightarrow \infty$ with $M_{n} / \sqrt{\log n} \rightarrow \infty$,

$$
\mathbb{E}_{\boldsymbol{\theta}_{0}} \Pi_{a}\left(n^{\beta /(2 \beta+1)}\left\|\boldsymbol{\theta}-\boldsymbol{\theta}_{0}\right\|>M_{n} \mid \mathbf{X}\right) \rightarrow 0
$$
as $n \rightarrow \infty$.

The applicability of Theorem 3.1 is limited due to the requirement to know $\beta$. As mentioned in the introduction, Bayesian procedures offer a natural framework for adaptive inference by placing appropriate priors on key hyperparameters. Indeed, we show that the proposed exponential-variance priors lead to minimax adaptive posterior convergence rates over all possible values of $\beta$ when $a$ is endowed with a suitable prior. Recall that the approach in Belitser \& Ghosal [1] leads to adaptation only over a discrete set of $\beta$-values.

For a prior distribution on $a$ having a density $h$ on $(0, \infty)$, we denote the following hierarchical prior on $\boldsymbol{\theta}$

$$
\boldsymbol{\theta} \mid a \sim \Pi_{a}, \quad a \sim h
$$

by $\Pi$, so that for any Borel set $A, \Pi(A)=\int \Pi_{a}(A) h(a) d a$. The corresponding posterior is denoted by $\Pi(\cdot \mid \mathbf{X})$. A fundamental object in studying posterior contraction rates along the lines of the general theory developed in [8] is the prior concentration, i.e., the probability assigned by the prior distribution to small neighborhoods of the true parameter. In the following Lemma 3.1, we develop a non-asymptotic prior concentration bound for the hierarchical prior П. The proof of Lemma 3.1 can be found in the supplemental document.

Lemma 3.1. Let $\boldsymbol{\theta}_{0} \in \Theta_{\beta}$. Consider the hierarchical prior $\Pi$ on $\boldsymbol{\theta}$ given by $\boldsymbol{\theta} \mid a \sim \Pi_{a}, a \sim h$, where $\Pi_{a}$ is as in (4). There exists positive constants $c_{1}, c_{2}, c_{3}$ such that for any $0<\epsilon<1$,

$\Pi\left(\left\|\boldsymbol{\theta}-\boldsymbol{\theta}_{0}\right\|<\epsilon\right) \geq c_{1} \exp \left\{-c_{2} \epsilon^{-1 / \beta}\left\{\log \left(c_{3} / \epsilon\right)\right\}^{2}\right\} P\left(\epsilon^{-1 / \beta} \leq a \leq 2 \epsilon^{-1 / \beta}\right)$.

The constants $c_{1}, c_{2}, c_{3}$ above depend on $\beta$ and $\left\|\boldsymbol{\theta}_{0}\right\|_{\beta}$, but not on $\epsilon$. 
For our adaptivity result, we require the prior density $h$ of $a$ to satisfy

$$
C_{1} x^{p} \exp \left(-D_{1} x \log ^{q} x\right) \leq h(x) \leq C_{2} x^{p} \exp \left(-D_{2} x \log ^{q} x\right),
$$

for positive constants $C_{1}, C_{2}, D_{1}, D_{2}$, non-negative constants $\mathrm{p}$, q, and every

105 condition (7). Given this condition, the second term in the right hand side of (6), $P\left(c_{1} \epsilon^{-1 / \beta} \leq a \leq c_{2} \epsilon^{-1 / \beta}\right)$, is of the same order as the first term, a fact which we use in the proof of our main result below. The proof can be found in the supplemental document.

as $n \rightarrow \infty$.

We finally state an auxiliary result which is used to prove Theorem 3.2 , the proof is deferred to the supplemental document.

Lemma 3.2. Assume the setting of Theorem 3.2 and recall the constant $q$ appearing in the bound for $h$ in (7). For any sequence of positive numbers $B_{n}$ such that $B_{n} /(\log n)^{2 \beta(2 \beta+1) \max \{2-q /(2 \beta), q /(2 \beta)\}} \rightarrow \infty$ as $n \rightarrow \infty$,

$$
\mathbb{E}_{\boldsymbol{\theta}_{0}} \Pi\left(\boldsymbol{\theta}:\|\boldsymbol{\theta}\|_{\beta}^{2}>B_{n} \mid \mathbf{X}\right) \rightarrow 0
$$

\subsection{Connection with rescaled Gaussian process priors}

Gaussian processes [11] are widely used as nonparametric priors on function spaces. In a series of seminal papers, van der Vaart \& van Zanten [9, 12, 10] studied for the first time posterior contraction rates in a variety of statistical models involving Gaussian process priors and showed that the reproducing kernel Hilbert space (RKHS) of a Gaussian process plays a key role in dictating the 
posterior convergence rate. See [13] for a review on aspects of RKHS related to posterior concentration.

The law of a mean zero Gaussian process is entirely specified by its covariance kernel $c(s, t)=\mathbb{E} W_{s} W_{t}$. A commonly used covariance kernel is the squared exponential kernel, given by $c(s, t)=\exp \left(-|s-t|^{2}\right)$. It is well known that the sample paths of a Gaussian process with the above kernel are infinitely smooth (in fact, analytic) and therefore not amenable for modeling less smooth functions. An influential idea of rescaled Gaussian processes was introduced in van der Vaart \& van Zanten [9]. In the original formulation, a rescaled Gaussian process $W_{t}^{a}=\left\{W_{a t}: t \in[0,1]\right\}$ stretches the sample paths of a smooth Gaussian process $W_{t}$ using a scalar parameter $a$, with the intuition that larger values of $a$ would lead to traversing the sample path of the unscaled process on the larger interval $[0, a]$, thereby incurring more roughness. Indeed, it was shown that the choice $a \equiv a_{n}=n^{1 /(2 \beta+1)}$ leads to optimal rate of posterior convergence for $\beta$ smooth functions. van der Vaart \& van Zanten [10] extended the idea to achieve adaptive estimation of any $\beta$ smooth function by placing a prior $h$ on $a$ satisfying (7) (in case of univariate function estimation).

We shall now argue that the parameter $a$ in the exponential-variance prior proposed in this paper is intimately related to the rescaling parameter $a$ in a rescaled Gaussian process with a squared exponential kernel. To this end, we shall adopt a weight-space view [1] of Gaussian processes. Let $\left\{\psi_{i}\right\}$ denote a system of orthonormal eigenfunctions (with respect to some density function $\rho$ ) and $\left\{\lambda_{i}\right\}$ the associated non-negative eigenvalues of a kernel function $c(\cdot, \cdot)$, so that

$$
\int c(s, t) \psi_{i}(t) \rho(t) d t=\lambda_{i} \psi_{i}(s), \quad i=1,2, \ldots
$$

By Mercer's theorem (Theorem 4.2 in [1]), the covariance kernel can be expanded as

$$
c(s, t)=\sum_{i=1}^{\infty} \lambda_{i} \psi_{i}(s) \psi_{i}(t) .
$$

Defining $\tau_{i}=\sqrt{\lambda_{i}}$, the sequence $\boldsymbol{\tau}=\left(\tau_{1}, \tau_{2}, \ldots\right) \in \ell_{2}$. Further, by the Karhunen-Loève Theorem [14, a mean-zero Gaussian process $W_{t}$ with covari- 
ance kernel $c(\cdot, \cdot)$ can be expressed as

$$
W_{t}=\sum_{i=1}^{\infty} \tau_{i} Z_{i} \psi_{i}(t)
$$

where $Z_{i}$ s are i.i.d. $\mathrm{N}(0,1)$ and the convergence is in $L_{2}(\rho)$.

An expansion as in the above display suggests a prior on $\ell_{2}$ as $\theta_{i}=\tau_{i} Z_{i} \sim$ $\mathrm{N}\left(0, \tau_{i}^{2}\right)$. For the squared exponential kernel $c_{a}(s, t)=e^{-a^{2}|s-t|^{2}}$ with rescaling parameter $a$ and a Gaussian weight function $\rho(x)=\sqrt{2 b / \pi} e^{-2 b x^{2}}$, the eigenvalues $\lambda_{i}$ and eigenfunctions $\psi_{i}$ are given by [1]

$\lambda_{i}=\left(\frac{2 b}{b+a^{2}+c}\right)^{1 / 2}\left(\frac{a^{2}}{b+a^{2}+c}\right)^{i-1}, \quad \psi_{i}(x)=\frac{(c / b)^{1 / 4}}{\sqrt{2^{i-1}(i-1) !}} e^{-(c-b) x^{2}} H_{i-1}(\sqrt{2 c} x)$,

where $c=\sqrt{b^{2}+2 b a^{2}}$ and $H_{i}$ are the physicist's Hermite polynomials. Observe that although the eigenvalues $\lambda_{i}$ decrease exponentially fast with $i$ for a fixed value of $a$, the decay rate slows down with increasing $a$. Further, fixing $b$ to be a constant,

$$
\lambda_{i} \asymp \frac{1}{a} e^{-(i-1) \log \left\{\left(b+a^{2}+c\right) / a^{2}\right\}} .
$$

Replacing $(i-1)$ by $i$ and $\log \left\{\left(b+a^{2}+c\right) / a^{2}\right\} \asymp \log (1+1 / a)$ by $1 / a$ suggests choosing the prior variance of $\theta_{i}$ as $a^{-1} e^{-i / a}$ in the proposed exponentialvariance prior.

The above connection may be useful in obtaining rates of convergence for Gaussian process priors using a more direct approach bypassing the involved calculations with the RKHS 9, 10. Further, since we identify $\ell_{2}$ with $L_{2}(\rho)$, rates of convergence can be derived in the $L_{2}(\rho)$ norm instead of the usual empirical $L_{2}$ norm. However, it will require substantial work to make the connection explicit; we hope to report such results elsewhere.

\section{Discussion}

In this article, we have proposed a new class of priors which lead to adaptive estimation over Sobolev balls in the Gaussian sequence model. Very recently, a line of study is emerging where frequentist coverage properties of Bayesian procedures in nonparametric models are being investigated [15, 16]. Specifically, 
these papers focus on inverse problems in the sequence space and consider the usual power-variance priors on $\ell_{2}$. It would be interesting to study whether similar coverage results can be proven for the exponential-variance prior proposed here. Further, given the connection with Gaussian process priors, it would be of interest to extend the coverage results to function estimation settings.

\section{References}

[1] E. Belitser, S. Ghosal, Adaptive bayesian inference on the mean of an infinite-dimensional normal distribution, The Annals of Statistics 31 (2) (2003) 536-559.

[2] A. B. Tsybakov, Introduction to nonparametric estimation, Vol. 11, Springer.

[3] M. S. Pinsker, Optimal filtering of square-integrable signals in Gaussian noise, Problemy Peredachi Informatsii 16 (2) (1980) 52-68.

[4] C. Stone, Optimal global rates of convergence for nonparametric regression, The Annals of Statistics (1982) 1040-1053.

[5] S. Y. Efroimovich, M. S. Pinsker, Learning algorithm of non-parametric filtering, Autom. Remote Control (45) (1984) 1434-1440.

[6] I. Johnstone, Gaussian estimation: sequence and multiresolution models, Unpublished manuscript. http://www-stat. stanford. edu/ ${ }^{\sim}$ imj.

175 [7] L. H. Zhao, Bayesian aspects of some nonparametric problems, Annals of statistics (2000) 532-552.

[8] S. Ghosal, J. K. Ghosh, A. W. Van Der Vaart, Convergence rates of posterior distributions, Annals of Statistics 28 (2) (2000) 500-531.

[9] A. van der Vaart, J. van Zanten, Bayesian inference with rescaled Gaussian process priors, Electronic Journal of Statistics 1 (2007) 433-448. 
[10] A. van der Vaart, J. van Zanten, Adaptive Bayesian estimation using a Gaussian random field with inverse Gamma bandwidth, The Annals of Statistics 37 (5B) (2009) 2655-2675.

[11] C. Rasmussen, Gaussian processes in machine learning, Advanced Lectures on Machine Learning (2004) 63-71.

[12] A. van der Vaart, J. van Zanten, Rates of contraction of posterior distributions based on gaussian process priors, The Annals of Statistics 36 (3) (2008) 1435-1463.

[13] A. van der Vaart, J. van Zanten, Reproducing kernel Hilbert spaces of Gaussian priors, IMS Collections 3 (2008) 200-222.

[14] R. J. Adler, An introduction to continuity, extrema, and related topics for general gaussian processes, Lecture Notes-Monograph Series (1990) i-155.

[15] B. Knapik, A. van Der Vaart, J. Van Zanten, et al., Bayesian inverse problems with gaussian priors, The Annals of Statistics 39 (5) (2011) 2626-2657.

[16] B. Szabo, A. van der Vaart, H. van Zanten, Frequentist coverage of adaptive nonparametric bayesian credible sets, Annals of Statistics, to appear.

[17] P. Natalini, B. Palumbo, Inequalities for the incomplete gamma function, Math. Inequal. Appl 3 (1) (2000) 69-77.

[18] S. Ghosal, A. Van Der Vaart, et al., Convergence rates of posterior distributions for noniid observations, The Annals of Statistics 35 (1) (2007) $192-223$.

[19] A. Barron, M. J. Schervish, L. Wasserman, et al., The consistency of posterior distributions in nonparametric problems, The Annals of Statistics 27 (2) (1999) 536-561. 